\title{
THE SPACE OF MINIMAL ANNULI BOUNDED BY AN EXTREMAL PAIR OF PLANAR CURVES
}

\author{
WILLIAM H. MEEKS, III AND BRIAN WHITE
}

\section{INTRODUCTION}

In 1956 Shiffman [14] proved that every minimally immersed annulus in $\mathbb{R}^{3}$ bounded by convex curves in parallel planes is embedded. He proved this theorem by showing that the minimal annulus was foliated by convex curves in parallel planes. We are able to prove a related embeddedness theorem for extremal convex planar curves. Recall that a subset of $\mathbb{R}^{3}$ is extremal if it is contained on the boundary of its convex hull. We will call a pair of convex curves extremal if their union is extremal.

Theorem 0.1. Suppose $\Gamma$ is an extremal pair of disjoint smooth convex planar curves in $\mathbb{R}^{3}$. If $f: A \rightarrow \mathbb{R}^{3}$ is a branched minimally immersed annulus with boundary $\Gamma$, then $f$ is an embedding.

We shall call a compact minimal surface $M$ stable if, with respect to any nontrivial normal variation fixing the boundary, the second derivative of area is positive. If the second derivative of area is negative for some variation, then $M$ is called unstable. If $M$ is neither stable nor unstable, we will call it almost-stable.

The main theorem of Meeks-White in [10] gives a precise description of the collection of minimal annuli bounded by a pair of convex curves in parallel

The research of the first author described in this paper was supported by research grant DE-FG02-86ER250125 of the Applied Mathematical Science subprogram of Office of Energy Research, U.S. Department of Energy, and National Science Foundation grant DMS-8900285.

The research of the second author was funded by National Science Foundation grants DMS-8553231 (PYI) and DMS-8703537. 
planes, in terms of the index of the Jacobi operator (stability operator) of the minimal annulus. The next theorem gives a complete generalization of this theorem to the case of an extremal pair of convex planar curves.

Theorem 0.2. If $\Gamma$ is an extremal pair of smooth disjoint convex curves in distinct planes, then exactly one of the following holds:

(1) $\Gamma$ is not the boundary of any connected compact minimal surface, with or without branch points.

(2) $\Gamma$ is the boundary of exactly one minimal annulus and this annulus is almost-stable. In this case, $\Gamma$ bounds no other connected branched minimal surfaces.

(3) $\Gamma$ is the boundary of exactly two minimal annuli, one stable and one unstable.

In Statement (3) of Theorem 0.2, we do not know (even for boundary curves in parallel planes) whether it is possible for $\Gamma$ to bound other minimal surfaces of higher genus; we believe that such higher genus examples do not exist. The extremal hypothesis in the above theorem is necessary, since we will construct an example of two unlinked convex planar curves that are the boundary of at least two stable minimal annuli (see Section 3).

The next theorem demonstrates the existence of an immersed minimal annulus $A$ with extremal disjoint planar boundary curves and such that $A$ is not embedded. This example is constructed in Section 4.

Theorem 0.3. For every $\varepsilon>0$ there exists a smooth stable minimally immersed annulus $f_{\varepsilon}: A \rightarrow \mathbb{R}^{3}$ such that:

(1) $f_{\varepsilon} \mid \partial A$ is one-to-one and the image boundary curves are a pair of Jordan curves in parallel planes;

(2) $f_{\varepsilon}$ is not one-to-one;

(3) the total curvature of each component of $f_{\varepsilon}(\partial A)$ is less than $4 \pi+\varepsilon$;

(4) $f_{\varepsilon}(A)$ is invariant under reflection in the middle plane that separates the components of $f_{\varepsilon}(\partial A)$ and in a plane orthogonal to the middle plane.

In light of Theorems 0.1 and 0.3 it is natural to ask: 
QUESTION 0.1. Suppose $\Gamma$ is a pair of simple closed curves on parallel planes, each component of which has total curvature less than $4 \pi$. Is every minimally immersed annulus with boundary $\Gamma$ embedded?

It follows easily from Theorem 0.3 and the maximum principle that the space $\widetilde{S}$ of smooth immersed minimal annuli with embedded pairwise disjoint extremal planar boundary curves is not a path connected space. However, in Section 5 we are able to prove:

Theorem 0.4. If $S \subset \widetilde{S}$ is the subspace of embedded minimal annuli, then $S$ is path connected.

The above theorem is a simple consequence of the more general result, Theorem 5.1, that the space $\mathcal{M}$ of embedded minimal annuli whose boundary curves are contained in distinct horizontal planes is a contractible space.

The results in this paper were announced in [9].

\section{The PRoOF of Theorem 0.1}

We begin by fixing some notation. In this section we will consider branched minimal immersions $f: A \rightarrow \mathbb{R}^{3}$ of an annulus $A$ such that $f(\partial A)=\Gamma=$ $\left\{\gamma_{1}, \gamma_{2}\right\}$ is an extremal pair of disjoint convex curves in distinct non-parallel planes. Without loss of generality we may assume that $\gamma_{1} \subset H=\left\{\left(x_{1}, x_{2}, 0\right) \mid\right.$ $\left.x_{1} \geq 0\right\}$ and $\gamma_{2} \subset H(\theta)$ where $H(\theta)$ is obtained by rotating $H$ around the $x_{2}$-axis by an angle $\theta, 0<\theta<\pi$, and $H(\theta)$ is a nonnegative graph over its projection on the $\left(x_{1}, x_{2}\right)$-plane. Let $W(\theta)$ be the convex wedge with boundary $H \cup H(\theta)$ and we will consider $f: A \rightarrow \mathbb{R}^{3}$ to have $W(\theta)$ as its range; i.e., $f: A \rightarrow W(\theta)$.

Definition 1.1. A branched minimal immersion $f: A \rightarrow W(\theta)$ is called $e x$ tremal if $f(\partial A)=\Gamma=\left\{\gamma_{1}, \gamma_{2}\right\}$ consists of two smooth disjoint simple closed curves with $f\left(\gamma_{1}\right) \subset H$ and $f\left(\gamma_{2}\right) \subset H(\theta)$.

Our first result is

Lemma 1.1. If $f: A \rightarrow W(\theta)$ is an extremal branched minimal immersion, then $f$ is an immersion. Furthermore $f(A)$ intersects $H(\eta)$ transversally in a single immersed closed curve for $0 \leq \eta \leq \theta$. 
Proof. Since $\partial W(\theta)$ is convex, $f \mid \partial A$ is an immersion and hence $f$ is an immersion near $\partial A$ (see Theorem 2 in [12]). Since $f(\operatorname{Int}(A)) \subset \operatorname{Int}(W(\theta))$ by the convex hull property, we may assume, after possibly replacing $A$ by its image in a slightly smaller subwedge of $W(\theta)$, that $A$ is disjoint from the line $H \cap H(\theta)$. If $p \in A$ is a branch point, then $p \in H(\tau)$ for some $\tau \in(0, \theta)$. In this case $\Delta=f^{-1}(H(\tau))$ is a compact singular one-dimensional analytic subset of $\operatorname{Int}(A)$. But such a subset separates $A$ into at least three components, one whose boundary is disjoint from $\partial A$. Since the boundary of this component under composition with $f$ is contained in the plane $H(\tau)$, the maximum principle implies the component is contained in $H(\tau)$, a contradiction. This contradiction proves the first statement in the lemma. The second statement in the lemma follows from a similar argument.

Lemma 1.2. Suppose $f: A \rightarrow W(\theta)$ is an extremal minimal immersion of $A$. If the components of $f(\partial A)$ are strictly convex, then the Gauss map $g: A \rightarrow \mathbb{S}^{2}$ is one-to-one. If $f(\partial A)$ is convex, then $g: \operatorname{Int}(A) \rightarrow \mathbb{S}^{2}$ is one-to-one.

Proof. We will identify $\mathbb{S}^{2}$ with $\mathbb{C} \cup\{\infty\}$ under stereographic projection. Let $S$ be a slab in $\mathbb{R}^{3}$ with boundary planes parallel to the $\left(x_{1}, x_{2}\right)$-plane. In the case $h: E \rightarrow S$ is a minimal immersion of an annulus $E$ and $h(\partial E)$ are convex curves in the boundary planes $\partial S$, it was proved in [10] that the Gauss map $g: \operatorname{Int}(E) \rightarrow \mathbb{C} \cup\{\infty\}$ is one-to-one and $g: E \rightarrow \mathbb{S}^{2}$ is one-to-one when $h(\partial E)$ is strictly convex. We wish to generalize this result to the following.

Assertion 1.1. Suppose $E$ in $S$ is an immersed annulus of nonpositive Gaussian curvature with smooth embedded boundary curves in $\partial S$ and such that the Gauss map $g: E \rightarrow \mathbb{C} \cup\{\infty\}$ is a branched covering map. If $\partial E$ is a pair of strictly convex curves, then $g$ is one-to-one. If $\partial E$ is a pair of convex curves, then $g \mid \operatorname{Int}(E)$ is one-to-one.

Proof. The Annulus Lemma in [8] shows that every plane $P$ between the boundary planes of $S$ intersects $E$ transversely in a single immersed curve. In particular $g: E \rightarrow \mathbb{C} \cup\{\infty\}$ avoids the values $0, \infty$. First consider the case when $\partial E=\partial_{1} \cup \partial_{2}$ is a pair of strictly convex curves. In this case $\partial_{1}$, as well as $\partial_{2}$, can be naturally parametrized by $\theta \in \mathbb{S}^{1}$ by considering $\partial_{1}$ to be 
parametrized by its outward pointing unit normal vector in $\partial S$. With this orientation of $E, g$ has the property that $\arg \left(g\left(\partial_{1}(\theta)\right)\right)=\theta$ and so $g$ restricted to each boundary component of $\partial E$ yields an embedded curve in $\mathbb{C}-\{0\}$ that has nonzero winding number. Since $g \mid \operatorname{Int}(E)$ is an open mapping, elementary convering space theory implies $g$ is one-to-one. If $\partial E$ is a pair of convex curves, then a simple perturbation argument of the strictly convex case proves $g \mid \operatorname{Int}(E)$ is one-to-one.

Suppose for the moment that $f: A \rightarrow W(\theta)$ does not intersect the line $H \cap H(\theta)$ and that the curves in $f(\partial A)$ are uniformly convex. Let $P_{t}, 0 \leq t<$ 1 , be a continuous path of projective transformation taking $W(\theta)$ to convex wedges in $\mathbb{R}^{3}$ and such that the $P_{t}$ converge to a projective transformation $P_{1}$ that takes $W(\theta)-(H \cap H(\theta))$ to a slab $S$. Here $P_{0}$ is the identity map. Since $P_{1} \circ f: A \rightarrow S$ satisfies the hypotheses of the annulus in the previous paragraph, the Gauss map $g_{1}$ of $P_{1} \circ f$ is one-to-one on $\operatorname{Int}(A)$ and one-to-one on $A$. Let $g_{t}$ be the Gauss map of $P_{t} \circ f$. Since $g_{t} \mid \operatorname{Int}(A)$ is an open mapping and $g_{1} \mid \partial A$ is a one-to-one immersion, it is clear that there cannot be a largest $t$ such that $g_{t}$ is not one-to-one. Thus $g_{0}$ is one-to-one.

If $f(\partial A) \cap H \cap H(\theta) \neq \emptyset$ or the curves in $f(\partial A)$ are not uniformly convex, a perturbation argument shows the similar statements hold for $f$. This completes the proof of the lemma.

Corollary 1.3. An extremal minimal immersion $f: A \rightarrow W(\theta)$ with convex boundary curves has index 0 or 1 . Furthermore, if the annulus has index 1, then it does not have a Jacobi vectorfield.

Proof. By Lemma 1.2 the Gauss map $g: \operatorname{Int}(A) \rightarrow \mathbb{S}^{2}$ is one-to-one. A wellknown theorem of Schwarz states that an eigenfunction (with zero boundary values) of the stability (or Jacobi) operator $J$ of a compact orientable minimal surface $M$ can be identified with an eigenfunction of $\Delta+2$ on $\mathbb{S}^{2}$ for $g(\operatorname{Int} M)$, when $g$ is one-to-one on $\operatorname{Int}(M)$ (see [2] for a generalized version of Schwarz's theorem). Since the number of linearly independent eigenfunctions of $\Delta+2$ with eigenvalue less than or equal to zero is at most 1 on a proper subdomain of $\mathbb{S}^{2}, J$ can have at most one eigenfunction with eigenvalue less than or equal to zero. 
Remark 1.1. Actually Corollary 1.3 holds for minimal annuli bounded by convex planar curves not necessarily extremal. See Remark 2.2 in [10].

Proposition 1.4. Let $\Gamma_{t}$ be a one-parameter family of convex curves in $H \cup$ $H(\theta)$ such that

(1) $\Gamma_{0}$ bounds an almost-stable extremal minimal annulus $A$;

(2) The initial velocity vectorfield of $\Gamma_{t}$ at $t=0$ is a outward pointing normal vectorfield that is not identically zero.

Then there is an $\varepsilon>0$ such that for $t \in(-\varepsilon, 0), \Gamma_{t}$ bounds no minimal annulus near $A$ and for $t \in(0, \varepsilon), \Gamma_{t}$ bounds exactly two annuli, one stable and one unstable, near $A$. Furthermore, given an almost-stable extremal annulus $A$ with convex boundary, then there exists such a one-parameter family $\Gamma_{t}$.

Proof. Let $\nu$ be the unit normal vectorfield on $A$ that is outward pointing along $\partial A$. By Corollary $1.3, A$ has nullity 1 and index 0 , so it has a Jacobi field $u(x) \nu(x)$ with $u \mid \partial A=0$ and $u>0$ on $\operatorname{Int}(A)$. For $x \in \partial A$, let

$$
v(x)=\left(\nabla_{T(x)} u(x)\right) \nu(x),
$$

where $T(x)$ is a unit vector perpendicular to $\partial A$ pointing into $A$. By the boundary maximum principle, $v(x)$ is a strictly positive multiple of $\nu(x)$ for all $x \in \partial A$.

Now let $w$ be the initial velocity vectorfield for $\Gamma_{t}$ at $t=0$. Note that $w(x) \cdot v(x) \geq 0$ everywhere and is not identically 0 . Thus

$$
\int_{\partial A} w(x) \cdot v(x)>0 \text {. }
$$

This is precisely the transversality condition in [16] that guarantees that

$$
U=\left\{\operatorname{minimal} \widetilde{A} \mid \partial \widetilde{A}=\Gamma_{t} \text { for some } t \text { near } 0\right\}
$$

is a smooth curve.

(In more detail, transversality means ([16, Theorem $3.3(2,3)])$ that $w$ does not extend to a Jacobi field on $A$. But if we extend $w$ to be a vectorfield on $A$, then by Green's formula ([16, 1.4(2)]),

$$
\int_{A} J w \cdot u=\int_{A} w \cdot J u+\int_{\partial A}\left(w \cdot \frac{\partial u}{\partial n}-\frac{\partial w}{\partial n} \cdot u\right)=\int_{\partial A} w \cdot v>0
$$


where $J$ is the Jacobi operator. Thus $J w \neq 0$.)

Let $\left\{f_{s}: A \rightarrow W(\theta) \mid-\delta<s<\delta\right\}$ be a smooth parametrization of $U$ with $f_{0}(A)=A$. Now $\left.\frac{\partial}{\partial s}\right|_{s=0} f_{s}=Z(x)$ is a Jacobi vectorfield on $A$. Let $z(x)=Z(x) \cdot \nu(x)$. Since $f_{s}$ is a smooth parametrization of $U, z(x)$ is not identically zero. Note that since the $\left\{\Gamma_{t}\right\}$ are nested, $z \mid \partial A$ does not change sign. Since no proper subdomain of $A$ has zero as a smallest eigenvalue of $J, z$ does not change sign. The same eigenvalue argument shows that for all $t, t z(x)+u(x)$ does not change sign in $A$, which implies

$$
z(x)=c u(x) .
$$

Without loss of generality we may assume that $c=1$.

Let

$$
\varphi_{s}(x)=x+s Z(x) .
$$

Let $G\left[f_{s}\right]$ and $G\left[\varphi_{s}\right]$ denote the Gauss maps of $f_{s}$ and $\varphi_{s}$, respectively. Note that for a fixed $x \in \partial A, \frac{d}{d s} G\left[\varphi_{s}\right](x)$ is a tangent vector to $\mathbb{S}^{2}$ at $G\left[f_{0}(x)\right]$ that points into $G\left[f_{0}(A)\right]$. Then the same is true of $\frac{d}{d s} G\left[f_{s}(x)\right]$, since $\left.\frac{d}{d s}\right|_{s=0} f_{s}=$ $\left.\frac{d}{d s}\right|_{s=0} \varphi_{s}$. Thus for small positive $s$,

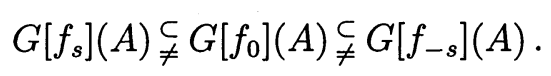

In particular, the first eigenvalue of $\Delta+2$ for the domain $G\left[f_{s}\right](A)$ is positive and the first eigenvalue of $G\left[f_{-s}\right](A)$ is negative. Since by Lemma 1.2 and Schwarz's theorem we can identify the stability operator of $f_{s}(A)$ with $\Delta+2$ on $G\left[f_{s}\right](A) \subset \mathbb{S}^{2}, f_{s}(A)$ is stable and $f_{-s}(A)$ is unstable.

Note that since the $\left\{\Gamma_{t}\right\}$ are nested,

$$
\left(\frac{\partial}{\partial s} f_{s}\right) \cdot G\left[f_{s}\right]
$$

does not change sign on $\partial A$. Hence for $s>0$, the stability of $F_{s}(A)$ implies that (1.1) does not change sign. The maximum principle then implies that (1.1) is strictly positive on the interior of $A$. Thus, the surfaces $\left\{f_{s}(A) \mid s>0\right\}$ locally foliate a one-sided neighborhood of $A$.

Since $\left.\frac{\partial}{\partial s}\right|_{s=0} f_{s} \cdot \nu=u>0$, these surface lie on the outside of $A$. Since the index of $f_{s}(A)$ changes by 1 at $s=0$, the projection of the curve $\left\{f_{s}(A) \mid\right.$ 
$-\delta<s<\delta\}$ to $\left\{\Gamma_{t} \mid-\varepsilon<t<\varepsilon\right\}$ folds at $s=0$ according to section 5.3 of [16]. Hence the $f_{s}(\partial A)$ and $f_{-s}(\partial A)$ lie on the same side of $\partial A$, namely the outside. This proves the first statement of the proposition.

(If it is not clear that the arguments in the preceding two paragraphs hold for immersions, one can reduce to the case of embedded annuli as follows. Since $f_{0}: A \rightarrow W(\theta)$ is an immersion, choose a $\delta>0$ and a submersion $\Pi: A \times[-\delta, \delta] \rightarrow W(\theta)$ so that $\Pi(\partial A \times[-\delta, \delta]) \subset \partial W(\theta)$ and $f_{0}=\Pi \circ i$ where $i(a)=(a, 0)$. Consider $A \times[-\delta, \delta]$ to be a flat three-manifold in the metric pulled back by $\Pi$. Note that for small $s, f_{s}$ lifts to an isometric minimal embedding $\widetilde{f}_{s}: A \rightarrow A \times[-\delta, \delta]$. By applying this lifting technique, we may reduce the proof to the case where $f_{0}$ is an embedding.)

We now justify the last assertion in the statement of the proposition. Deform $\partial A$ along an outward pointing vectorfield that vanishes except on a uniformly convex arc of $\partial A$ that is disjoint from the line $H \cap H(\theta)$. Such a deformation $\Gamma_{t}$ works.

Proof of Theorem 0.1. Let $\mathcal{M}$ denote the space of minimally immersed annuli whose boundary curves are contained in planes and let $p: \mathcal{M} \rightarrow \mathcal{C}$ be the natural projection where $\mathcal{C}$ is the associated space of boundary curves. Note that an extremal $f: A \rightarrow W(\theta)$ with convex boundary curves can be thought of as having its image in $\mathcal{M}$. Since such an annulus is unbranched (Lemma 1.1) and has total curvature less than $4 \pi$ in absolute value, the compactness theorems of Anderson [1] or White [15] imply that if $I$ is a compact collection of pairs of convex curves in $\mathcal{C}$ that are the boundary curves of extremal minimal immersions, then $p^{-1}(I)$ is compact.

Suppose that there exists an $f: A \rightarrow W(\theta)$ that is an extremal minimal immersion with convex boundary curves and $f$ is not one-to-one. We assert: we can choose $f$ so that $f(\partial A) \cap H \cap H(\theta)=\emptyset, f(\partial A)$ are strictly convex curves, and $f(\partial A)=\left\{\gamma_{1}, \gamma_{2}\right\}$ is a regular value of $p: \mathcal{M} \rightarrow \mathcal{C}$.

By Proposition 2.1 we can perturb $f$ to a nonembedded example $f_{1}: A \rightarrow$ $W(\theta)$ where $F_{1}(\partial A)$ is a regular value of $p: \mathcal{M} \rightarrow \mathcal{C}$. Since $f_{1}$ is a regular point of $p$, it can be moved slightly to another nonembedded minimal annulus, $f_{2}: A \rightarrow W(\theta)$, such that $f_{2}(\partial A) \cap H \cap H(\theta)=\emptyset, f_{2}(\partial A)$ are strictly convex 
curves and $f_{2}(\partial A)$ is a regular value of $p$. Replacing $f$ with $f_{2}$, we have proved our assertion. Assume now that $f$ satisfies the assertion.

Let $\alpha:[0,1] \rightarrow \mathcal{C}$ be a path that is in general position with respect to $p$ and such that

(1) $\alpha(0)=f(\partial A)$;

(2) $\{\alpha(t) \cap H\}$ is a foliation of an annulus $E$ on $H$ by strictly convex curves and with inner curve $\alpha(0) \cap H$;

(3) $\{\alpha(t) \cap H(\theta)\}$ is a foliation of an annulus $E(\theta)$ on $H(\theta)$ by strictly convex curves and with inner curve $\alpha(0) \cap H(\theta)$;

(4) $\alpha(t)=(\partial E \cup \partial E(\theta))-\alpha(0)$ is a pair of round circles that is invariant under reflection in the halfplane $H(\theta / 2)$.

The existence of an $\alpha$ satisfying properties (1)-(4) is clear. Theorem 2 in [13] implies that any compact minimal surface with boundary $\alpha(1)$ must be embedded, and invariant under reflection in $H(\theta / 2)$. So we must also have:

(5) Every minimal annulus in $p^{-1}(\alpha(1))$ is embedded.

Since $\alpha$ is in general position with respect to $p, W=p^{-1}(\alpha[0,1])$ is a smooth compact one-dimensional submanifold of $\mathcal{M}$. The maximum principle implies that every component of $T$ consists entirely of embedded or of nonembedded examples. Consider the component $T(f)$ of $T$ that contains $f$ in its boundary. Since the other boundary point $\tilde{f}$ of $T(f)$ corresponds to a nonembedded minimal annulus, the boundary of $\tilde{f}$ is also $\alpha(0)$. Thus by viewing $T(f)$ as a multivalued function over $\alpha[0,1]$, we see that $T(f)$ has an odd number of folds.

In the interval $T(f)$ consider the closest point $f^{\prime}$ to $f$ where $T(f)$ folds a first time. Suppose $p\left(f^{\prime}\right)=\alpha\left(t_{0}\right)$. Since $\alpha(t)$ is an outward variation of $\alpha\left(t_{0}\right)$ near $t_{0}$, Proposition 2.1 implies that $T(f)$ cannot fold over at $\alpha\left(t_{0}\right)$. This contradiction proves that $f$ must be embedded.

\section{The PROOF OF THEOREM 0.2}

Lemma 2.1. Suppose $\Delta=\{\alpha, \beta\}$ is a pair of smooth pairwise-disjoint simple closed curves with $\alpha \subset H$ and $\beta \subset H(\theta)$. Let $D_{\alpha}$ and $D_{\beta}$ be the compact planar disks with $\partial D_{\alpha}=\alpha$ and $\partial D_{\beta}=\beta$. Suppose that there exists a connected 
branched minimal surface $\Sigma$ whose boundary is contained in $\mathcal{D}=D_{\alpha} \cup D_{\beta}$. Under these hypotheses there exists a unique embedded minimal annulus $\mathcal{A}$ with $\partial \mathcal{A}=\Delta$ and such that the following statements hold:

(1) Let $B$ be the compact region of $\mathbb{R}^{3}$ with boundary $\mathcal{A} \cup \mathcal{D}$. Then every compact branched minimal surface $M$ with $\partial M \subset \mathcal{D}$ is contained in $B$.

(2) If $M$ is a compact branched minimal surface with $\partial M \subset \mathcal{D}$ and $\operatorname{Int}(M)$ $\cap \partial B \neq \emptyset$, then $M=\mathcal{A}$.

(3) $\mathcal{A}$ is stable or almost-stable.

Proof. A slight variation of this lemma appears as Lemma 2.1 in [10] so we will only briefly sketch its proof here and refer the reader to our other paper for further details.

First note that $\alpha$ and $\beta$ are homotopic in the complement of $\Sigma$ in $W(\theta)$. By the Geometric Dehn Lemma [11] [12], $\alpha$ and $\beta$ bound a least-area embedded annulus in the closure of the unbounded component of $W(\theta)-\Sigma$. Since the set of embedded minimal annuli with boundary $\alpha \cup \beta$ is compact, there exists a minimal annulus $\mathcal{A}$ such that the volume of the ball $B$ with boundary $\mathcal{A} \cup \mathcal{D}$ is largest. If there exists a compact minimal surface $M$ with $\partial M \subset \mathcal{D}$ and $M$ is not contained in $B$, then using $M \cup \mathcal{A}$ as a barrier one obtains a least-area embedded minimal annulus $A$ with $\partial A=\Delta$ and $A$ lies outside $M \cup \mathcal{A}$. In this case the ball bounded by $A \cup \mathcal{D}$ has volume greater than $B$, a contradiction.

Part (2) follows from the maximum principle. Part (3) follows since $\mathcal{A}$ must have least-area outside itself, otherwise using $\mathcal{A}$ as a barrier one could construct a smaller area surface in $W(\theta)-B$. This completes the proof of the lemma up to proving uniqueness of $\mathcal{A}$. Uniqueness of $\mathcal{A}$ follows from statements 1 and 2 in the lemma.

Proof of Theorem 0.2. Suppose $f: A \rightarrow W(\theta)$ is an extremal minimal immersion with convex boundary. By Theorem $0.1 f$ is one-to-one and we will identify $A$ with its image in $W(\theta)$.

Assertion 2.1. Suppose $A$ is the unique minimal annulus with boundary $\partial A$. Then $A$ is almost-stable and $A$ is the unique compact branched minimal surface with boundary $\partial A$. 
Proof of Assertion 2.1. If $\partial A$ is a regular value of $p: \mathcal{M} \rightarrow \mathcal{C}$, then $\partial A$ bounds an even number of minimal annuli; in fact, the number of odd index minimal annuli with boundary $\partial A$ equals the number of minimal annuli of even index (see Theorem 2.1 in [17]). Hence $A$ must have a nonzero Jacobi vectorfield. But, by Lemma 2.1, $A=\mathcal{A}$ and so $A$ is almost-stable.

Suppose $\partial A$ is the boundary of some other compact connected branched minimal surface $M$. By Lemma $2.1 M$ is contained in the ball $B$ with boundary $A \cup \mathcal{D}$. For small $\varepsilon>0$ let $A(\varepsilon)$ denote the subannulus of $A$ obtained by intersecting $A$ with the wedge with boundary halfplanes $H(\varepsilon)$ and $H(\theta-\varepsilon)$. Note that $A(\varepsilon)$ is stable. The proof of Theorem 1.2 in [5] generalizes to our situation to show that for $\varepsilon>0, \partial A(\varepsilon)$ is also the boundary of an unstable or almost-stable minimal annulus $\widetilde{A}(\varepsilon)$ such that $\widetilde{A}(\varepsilon) \cap M \neq \emptyset$. However, by uniqueness of $A$, the $\widetilde{A}(\varepsilon)$ converge smoothly to $A$ as $\varepsilon \rightarrow 0$. Since $M$ is inside $\mathcal{A}$ and $M$ is never tangent to $A$ along $\partial A$ (by the boundary maximum principle), the smooth convergence of $\widetilde{A}(\varepsilon)$ to $A$ implies that there is a small compact neighborhood $N(\partial M) \subset M$ of $\partial M$ such that for $\varepsilon$ close to zero, $\widetilde{A}(\varepsilon) \cap N(\partial M)=\emptyset$. It follows that there exists a sequence $\varepsilon(i) \rightarrow 0$ and a sequence of points $p_{i} \in \widetilde{A}(\varepsilon(i)) \cap(M-N(\partial M))$ such that $p_{i} \rightarrow p \in A \cap$ $\overline{(M-N(\partial M)}$. Since $A \cap M=\partial A, p$ must be contained in $H$ or $H(\theta)$. But $p \in \overline{M-N(\partial M)}$, which is disjoint from $H \cup H(\theta)$. This contradiction proves the assertion.

Suppose $\partial A$ is not a regular value of $p: \mathcal{M} \rightarrow \mathcal{C}$ and $A$ is not the unique minimal annulus with boundary $\partial A$. Note that $A=\mathcal{A}$, defined in Lemma 2.1, or else $A \neq \mathcal{A}$ and in either case it follows that there is a minimal annulus $\widetilde{A}$ with $\widetilde{A} \cap A=\partial A$. Without loss of generality we may assume that $A$ is almost-stable. We now show that $\widetilde{A}$ is a regular value for $p$, which just means by Corollary 1.3 that $\widetilde{A}$ is not almost-stable. Let $B$ be the piecewise-smooth ball in $\mathbb{R}^{3}$ bounded by $A$ together with the two planar disks bounded by $\partial A$; similarily define the ball $\widetilde{B}$ for $\widetilde{A}$. Assume that $A$ and $\widetilde{A}$ are oriented by the outward pointing normal to $B$ and $\widetilde{B}$, respectively. Let $g_{A}, g_{\widetilde{A}}$ denote the corresponding Gauss maps. First consider the case when $B \subset \widetilde{B}$. Since $g_{A} \mid \operatorname{Int}(A)$ and $g_{\widetilde{A}} \mid \operatorname{Int}(\widetilde{A})$ are diffeomorphisms with their respective images in 
$\mathbb{S}^{2}$ (Lemma 1.2) and $\widetilde{A}$ lies outside of $A$ along $\partial A=\partial \widetilde{A}$, a simple pointwise geometric comparison of $g_{A} \mid \partial A$ and $g_{\widetilde{A}} \mid \partial A$ yields $g_{\widetilde{A}}(\operatorname{Int}(\widetilde{A}))_{\neq}^{\complement} g_{A}(\operatorname{Int}(A))$. Since the first eigenvalue of the Jacobi operator of $A$ is zero, Schwarz's theorem (see the proof of Corollary 1.3) implies that the first eigenvalue of the Jacobi operator of $\widetilde{A}$ is positive. Under the hypothesis $\widetilde{B} \subset B$, the previous argument shows $g_{A}(\operatorname{Int}(A))_{\ddagger} g_{\widetilde{A}}(\operatorname{Int}(\widetilde{A}))$ and so, in this case, the first eigenvalue of the Jacobi operator of $\widetilde{A}$ is negative. Thus whether $B \subset \widetilde{B}$ or $\widetilde{B} \subset B, \widetilde{A}$ is not almost-stable; hence $\widetilde{A}$ is a regular point of $p$.

By Proposition 2.1 we can move $\partial A$ outward to a regular value $\widehat{\Gamma}$ of $p$ so that $A$ splits into a pair of close minimal annuli $A_{1}, A_{2}$. Since $\widetilde{A}$ is a regular point of $p$, we can also assume that $\widetilde{A}$ moves to a close annulus $A_{3}$. Hence, if Theorem 0.2 were to fail for $\Gamma$ not a regular value of $p$, then it fails for some $\Gamma$ a regular value of $p$.

Assertion 2.1 and the discussion in the previous two paragraphs show that we need only prove the theorem in the special case where $\partial A$ is a regular value of $p$. In this case $\partial A$ is the boundary of an even number of minimal annuli, half of which are stable and half of which are unstable. It remains to prove that $\partial A$ is the boundary of exactly two minimal annuli, or equivalently stated, $\partial A$ is the boundary of exactly one stable minimal annulus. Suppose $\partial A$ is the boundary of at least two stable minimal annuli $A_{1}, A_{2}$. By Lemma 2.1, we may choose $A_{1}$ to be $\mathcal{A}$ and so we may assume that $\operatorname{Int}\left(A_{1}\right) \cap \operatorname{Int}\left(A_{2}\right)=\emptyset$ and $A_{2}$ lies inside $A_{1}$.

Since $A_{1}$ and $A_{2}$ are minimal, the compact region $R$ with boundary $A_{1} \cup A_{2}$ is a solid torus. Note that $R \cap H(\theta / 2)$ is the unique minimal annulus in $R$ with the given boundary. It follows from this observation that the parity of the number of odd index minimal annuli in $R$ with boundary $\partial A_{1}$ is different from the parity of the number of even index minimal annuli in $R$ with boundary $\partial A_{1}$ (for a proof see Lemma 3 in [4]). In particular $\partial A_{1}$ is the boundary of an unstable minimal annulus $A_{3}$ in $R$. However, since $A_{3}$ lies outside $A_{2}$, the Gaussian image $g\left(\operatorname{Int}\left(A_{3}\right)\right) \varsubsetneqq g\left(\operatorname{Int}\left(A_{2}\right)\right)$. This proper inclusion contradicts the fact that the first eigenvalue of $\Delta+2$ of $g\left(\operatorname{Int}\left(A_{3}\right)\right)$ is smaller than the first eigenvalue of $\Delta+2$ for $g\left(\operatorname{Int}\left(A_{2}\right)\right)$. This contradiction completes the proof of Theorem 0.2 . 
The following theorem is a simple consequence of Theorem 0.2.

Theorem 2.2. Suppose $\Gamma$ is an extremal pair of smooth disjoint convex planar curves. Suppose $A$ is a minimal annulus in $\mathbb{R}^{3}$ with $\partial A=\Gamma$. Then every symmetry of $\Gamma$ extends to a symmetry of $A$.

Proof. Let $S: \mathbb{R}^{3} \rightarrow \mathbb{R}^{3}$ be a symmetry of $\Gamma$. Then $S(A)$ is another minimal annulus with boundary $\Gamma$ and $S(A)$ has the same index as $A$. Theorem 0.2 implies there is at most one minimal annulus with boundary $\Gamma$ of a fixed index and so $S(A)=A$.

\section{Minimal AnNuli BOUNDED By}

\section{A NONEXTREMAL PAIR OF CONVEX PLANAR CURVES}

In this section we will demonstrate by the construction of examples that Theorem 0.2 can not be generalized to the case of convex planar curves whose union is not extremal, even in the case of circles.

Consider the circle $\alpha$ in the $\left(x_{1}, x_{2}\right)$-plane of radius two and centered at the origin. For $\theta$ between 0 and $\pi$ let $\beta(\theta)$ be the circle of radius one centered at the origin in the $\left(x_{1}, x_{2}\right)$-plane that has been rotated clockwise around the positive $x_{2}$-axis by the angle $\theta$. Let $\alpha_{+}$denote the portion of $\alpha$ with nonnegative $x_{1^{-}}$ coordinate and let $\alpha_{-}=\overline{\alpha-\alpha_{+}}$. Let $\beta_{+}(\theta)$ be the portion of $\beta(\theta)$ with postive $x_{3}$-coordinate and $\beta_{-}(\theta)$ be $\overline{\beta(\theta)-\beta_{+}(\theta)}$. Let $\delta=\{(0, t, 0)|1 \leq| t \mid \leq 2\}$, $\gamma_{1}(\theta)=\delta \cup \alpha_{+} \cup \beta_{+}(\theta)$ and $\gamma_{2}(\theta)=\delta \cup \alpha_{-} \cup \beta_{+}(\theta)$.

Since $\gamma_{1}(\theta)$ and $\gamma_{2}(\theta)$ are each extremal, they bound, by the Geometric Dehn Lemma [11], least-area disks $D_{1}(\theta), D_{2}(\theta)$, respectively. Let $\widetilde{D}_{1}(\theta), \widetilde{D}_{2}(\theta)$ denote the disks obtained by rotating $D_{1}(\theta), D_{2}(\theta)$ by $\pi$ radians around the $x_{2}$-axis. Then $A_{1}(\theta)=D_{1}(\theta) \cup \widetilde{D}_{1}(\theta)$ and $A_{2}(\theta)=D_{2}(\theta) \cup \widetilde{D}_{2}(\theta)$ are distinct minimal annuli with boundary $\alpha \cup \beta(\theta)$. Note that $A_{1}(\pi / 2)$ can be obtained from $A_{2}(\pi / 2)$ by reflection in the $\left(x_{1}, x_{2}\right)$-plane. Hence $A_{1}(\pi / 2)$ and $A_{2}(\pi / 2)$ have the same index, which shows that Theorem 0.2 can not hold for $\alpha \cup \beta(\pi / 2)$. Actually $A_{1}(\pi / 2)$ and $A_{2}(\pi / 2)$ are stable and we believe $A_{1}(\theta)$ and $A_{2}(\theta)$ are stable for each $\theta, 0<\theta<\pi$.

One can, in a similar manner, construct two stable embedded minimal annuli with boundary two linked circles in orthogonal planes since orthogonal 


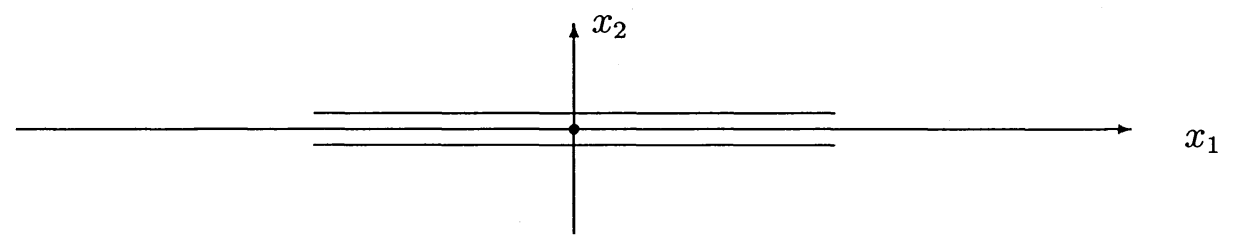

FIGURE 1. An $\varepsilon$-bone-shaped curve.

reflection in these planes fails to leave any minimal annulus bounding the circles invariant.

\section{NONEMBEDDED MINIMAL ANNULI BOUNDED BY JORDAN CURVES IN PARALLEL PLANES}

A frequently asked question in the classical theory of minimal surfaces is: Is every minimal annulus with boundary consisting of a pair of Jordan curves in parallel planes embedded? We will show that the answer to this question is no by constructing a family of counter-examples. This constriction will also prove Theorem 0.3 . The discussion of our new examples will be facilitated by the following definition.

Definition 4.1. For a fixed $\epsilon>0$, a smooth simple closed curve in the $\left(x_{1}, x_{2}\right)$-plane is called an $\varepsilon$-bone-shaped curve if it satisfies the following:

(1) $\gamma$ is invariant under reflection in the $x_{1}$-axis and the $x_{2}$-axis;

(2) $\gamma-\left(x_{1}\right.$-axis $)$ consists of two components $\gamma^{+}, \gamma^{-}$, each of which is a graph over their projection onto the $x_{1}$-axis and these graphs each have two inflexion points;

(3) The maximum curvature of $\gamma$ is less than $\varepsilon$;

(4) The total curvature of $\gamma$ is less than $2 \pi+\varepsilon$;

(5) The graph $\gamma^{+}$is the graph of the constant function $\varepsilon$ for $\left|x_{1}\right| \leq 1 / \varepsilon$ and obtains a height of at least 10 somewhere outside this interval. 
Proof of Theorem 0.4. For any $\varepsilon>0$, the existence of an $\varepsilon$-bone-shaped curve is clear. Choose an $\varepsilon<\frac{1}{100}$ and let $\gamma$ be an $\varepsilon / 2$-bone-shaped curve. Let $\alpha=\gamma+(0,0,1)$ and $\beta=\gamma-(0,0,1)$ be vertical translates of $\gamma$ to the horizontal planes $P(1)$ and $P(-1)$ of heights \pm 1 , respectively. Let $D_{\alpha}, D_{\beta}$ denote the corresponding planar disks with boundary curves $\alpha, \beta$. Since $\alpha \cup \beta$ bounds some minimal annulus for $\varepsilon / 2<\frac{1}{100}$, it is the boundary of an "outermost" minimal annulus $\mathcal{A}$ such that the compact ball $B$ with boundary $\mathcal{A} \cup D_{\alpha} \cup$ $D_{\beta}$ contains all compact minimal surfaces in $\mathbb{R}^{3}$ whose boundary curve are contained in $D_{\alpha} \cup D_{\beta}$. (See Lemma 2.1 for the existence of $\mathcal{A}$.)

By using catenoid barriers it is easy to check that the part of $\mathcal{A}$ that lies outside the infinite vertical cylinder over $\gamma$ must stay a distance less than $\frac{1}{10}$ from this cylinder. It follows that the compact stable catenoid with axis parallel to the $x_{2}$-axis and with circle boundaries of radius one and with $x_{2^{-}}$ coordinates $\pm \frac{1}{4}$ must intersect $B$ in an annular component $T$.

Since $\varepsilon<\frac{1}{100}$, there exists compact stable catenoids $C_{1}, C_{2}$ and $C_{3}$ satisfying

(1) $\partial C_{i}$ are circles of radius two and $\partial C_{i} \subset \operatorname{Int}\left(D_{\alpha} \cup D_{\beta}\right)$;

(2) $C_{1}$ has $x_{1}$-coordinate less than $-1 / \varepsilon$ and is symmetric about the $\left(x_{1}, x_{3}\right)$ plane;

(3) $C_{2}$ has $x_{1}$-coordinate greater than $1 / \varepsilon$ and has positive $x_{2}$-coordinate;

(4) $C_{3}$ is the image under reflection in the $\left(x_{1}, x_{3}\right)$-plane of $C_{2}$.

Note that since $\mathcal{A}$ is outermost, $C_{i} \subset B$ and $C_{i} \cap \partial B=\partial C_{i}$.

We are now ready to describe the new example. First we define its boundary curves. Consider a curve as in Figure 2 having total curvature at most $4 \pi+$ $\varepsilon$. Note that this curve partially coincides with the $\varepsilon / 2$-bone-shaped curve $\gamma$ and is invariant under reflection in the $x_{1}$-axis. Let $\delta$ and $\sigma$ denote the vertical translates of this curve to $D_{\alpha}$ and $D_{\beta}$ and let $\Gamma=\{\delta, \sigma\}$. We wish to construct a stable non-embedded minimal annulus $A$ with $\partial A=\Gamma$ such that $A$ is invariant under reflection in the $\left(x_{1}, x_{2}\right)$ and $\left(x_{1}, x_{3}\right)$-planes. 


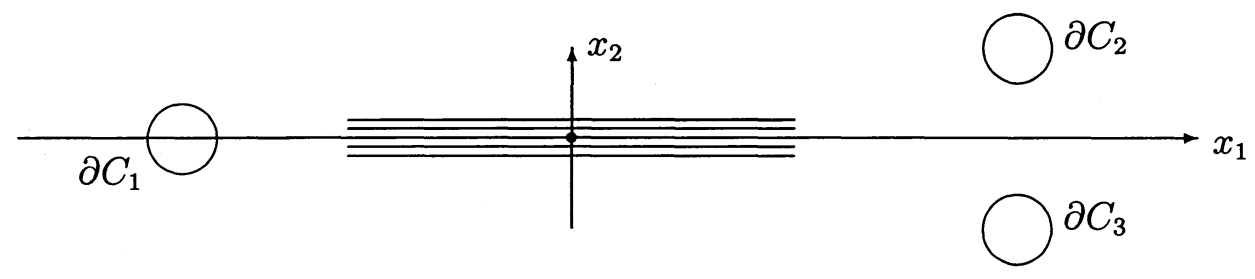

Figure 2: The projection of $\delta \cup \sigma, \partial C_{1}, \partial C_{2}, \partial C_{3}$ on the $\left(x_{1}, x_{2}\right)$-plane

Let $W$ denote the closure of the component of $B-\left(C_{2} \cup C_{3}\right)$ that contains $\mathcal{A}$. The fundamental group $\pi_{1}(W)$ is generated by one each of the boundary curves of $C_{2}$ and $C_{3}$. Consider the representation $\pi_{1}(W) \rightarrow \mathbb{Z}_{2}$ obtained by sending each of these generators to the generator of $\mathbb{Z}_{2}$. Let $p: \widetilde{W} \rightarrow W$ denote the 2-sheeted cover of $W$ corresponding to this representation. Let $Y$ denote the subdomain of $W$ with negative $x_{1}$-coordinates. Since $Y$ is a ball topologically, $p^{-1}(Y)$ consists of two ball components $Y_{1}, Y_{2}$. Let $\widetilde{\delta}$ denote the lift of $\delta$ to $\widetilde{W}$ such that the $\operatorname{arc} p\left(\widetilde{\delta} \cap Y_{1}\right)$ lies outside the $\operatorname{arc} p\left(\widetilde{\delta} \cap Y_{2}\right)$ in $Y$ (i.e. the minimum value of the $x_{1}$-coordinate of $p\left(\tilde{\delta} \cap Y_{1}\right)$ is less than the minimum value on $\left.p\left(\tilde{\delta} \cap Y_{2}\right)\right)$. Similarily choose the lift $\tilde{\sigma}$ of $\sigma$.

Let $\widetilde{T}$ denote the lift of $T$ to $\widetilde{W}$ such that $\widetilde{T} \cap Y_{1} \neq \emptyset$ and let $\widetilde{C}_{1}$ denote the lift of $C_{1}$ to $\widetilde{W}$ and such that $\widetilde{C}_{1} \subset Y_{2}$. Let $\widehat{W}$ denote the closure of the component of $\widetilde{W}-\left(\widetilde{T} \cup \widetilde{C}_{1}\right)$ that contains $\widetilde{\delta} \cup \widetilde{\sigma}$. Since $\partial \widehat{W}$ is a good barrier for solving leastarea problems (i.e. $\partial \widehat{W}$ has nonnegative mean curvature and interior angles less than $\pi$ ) and since $\widetilde{\delta}$ is homotopic to $\widetilde{\sigma}$ in $\widehat{W}$ but not homotopically trivial, the Geometric Dehn Lemma [11] [12] states that $\tilde{\delta} \cup \tilde{\sigma}$ is the boundary of an embedded least-area incompressible annulus $\widetilde{A}$ in $\widehat{W}$.

Since $\widetilde{A}$ is incompressible and $Y_{1}$ is simply connected, $\widetilde{A} \cap Y_{2}$ consists of two disks whose projections into $W$ are disjoint from $T$. Similarly $\tilde{A} \cap Y_{1}$ consists of a strip with part of its boundary in $\tilde{\delta}$ and part in $\widetilde{\sigma}$. It follows from this description of $\widetilde{A}$ that the projection $A=p(\widetilde{A})$ is not embedded. 
It remains only to prove that $A$ is invariant under reflection $R_{1}$ in the $\left(x_{1}, x_{2}\right)$-plane and reflection $R_{2}$ in the $\left(x_{1}, x_{3}\right)$-plane. Note that $R_{1}$ and $R_{2}$ lift to reflectional symmetries $\widetilde{R}_{1}, \widetilde{R}_{2}$ of $\widetilde{W}$, whose fixed point sets intersect $\widetilde{A}$ in nonempty subsets. Since $\widetilde{A}$ is incompressible, the version of the Geometric Dehn's Lemma for planar domains in [11] shows that the least-area annulus $\widetilde{R}_{i}(\widetilde{A})$ must equal the least-area annulus $\widetilde{A}$. This invariance property of $\widetilde{A}$ implies $A$ is invariant under $R_{1}$ and $R_{2}$.

\section{The topology of the SPACE OF EMBEDDED Minimal ANNUli}

The main theorem of this section is the following:

Theorem 5.1. The space $\mathcal{M}$ of compact embedded minimal annuli whose boundary curves are contained in distinct horizontal planes is contractible. In particular, $\mathcal{M}$ is a path connected space.

The idea of the proof is the following. In general, stable minimal surfaces depend smoothly on their boundaries, so that arbitrary small deformations of the boundary of a stable minimal surface extend to deformations of the surface (preserving minimality). Indeed, a one-parameter family of deformations of the boundary extends to a family of deformations of the surface until the surface ceases to be stable. In our case, we first intersect an initial annulus by thinner and thinner slabs until we get a ribbon-like minimal surface. This ribbon-like surface is so stable that we can deform its boundary curves to circles with the same axis without it becoming unstable. But then we must have deformed the surface itself to a catenoid. This shows that any annulus is $\mathcal{M}$ can be deformed into a catenoid. Likewise any compact family of annuli in $\mathcal{M}$ can be deformed until they are all the same catenoid. Thus $\mathcal{M}$ is contractible. To make the argument precise we use the following lemma:

Lemma 5.2. Let $P_{t}$ be the plane $\left\{(x, y, z) \in \mathbb{R}^{3} \mid z=t\right\}$. Let $\mathcal{C}$ be a compact set of $C^{2, \alpha}$ embedded curves in $P_{0}$ (with uniformly bounded $C^{2, \alpha}$ norms), and 
let $\Lambda>0$. There is an $\varepsilon>0$ such that if

$$
\begin{aligned}
h & \in(0, \varepsilon) \\
\gamma & \in \mathcal{C} \\
\gamma^{\prime} \subset P_{h}, \quad\left\|\gamma^{\prime}\right\|_{2, \alpha} & \leq \Lambda, \quad\left\|\gamma^{\prime}-\gamma\right\|_{0} \leq \Lambda h,
\end{aligned}
$$

then no unstable or almost-stable minimal annulus with boundary $\gamma \cup \gamma^{\prime}$ lies in an $\varepsilon$-neighborhood of $\gamma$.

Proof. Let $U_{r}(\gamma)$ denote the $r$-neighborhood of (the image of) $\gamma$. Choose $r>0$ such that for each $\gamma \in \mathcal{C}$ and $h \in(0, r), U_{h}(\gamma)$ is a regular tubular neighborhood with mean convex boundary (i.e., the mean curvature of $\partial U_{h}(\gamma)$ is everywhere nonzero and points into $\left.U_{h}(\gamma)\right)$.

Suppose the lemma is false. Then there are sequences $h_{i} \rightarrow 0, \gamma_{i}$, and $\gamma_{i}^{\prime}$ as in the statement of the lemma and non-stable minimal annuli $A_{i} \subset U_{r}\left(\gamma_{i}\right)$ with $\partial A_{i}=\gamma_{i} \cup \gamma_{i}^{\prime}$. Let $\rho_{i}$ be the smallest positive number such that $A_{i} \subset \bar{U}_{\rho_{i}}\left(\gamma_{i}\right)$. By the mean convexity and the maximum principle, $\partial U_{\rho_{i}}\left(\gamma_{i}\right)$ touches $A_{i}$ only at boundary points of $A_{i}$. In particular, it touches $\gamma_{i}^{\prime}$. Thus $\rho_{i} \leq h_{i} \sqrt{1+\Lambda^{2}}$, so

$$
A_{i} \subset \bar{U}_{h_{i} \sqrt{1+\Lambda^{2}}}\left(\gamma_{i}\right)
$$

Also note by the Gauss-Bonnet theorem that the total curvatures of the $A_{i}$ are uniformly bounded.

Let $p_{i} \in A_{i}$ be the point where the Gaussian curvature $K_{i}\left(p_{i}\right)=K_{A_{i}}\left(p_{i}\right)$ is greatest in absolute value. We claim that $\left|K_{i}\left(p_{i}\right)\right|^{1 / 2}=o\left(1 / h_{i}\right)$. For if not, we would have (after passing to a subsequence)

$$
\left|K_{i}\left(p_{i}\right)\right|^{1 / 2} h_{i} \geq \delta
$$

for some $\delta>0$. Now translate $A_{i}$ by $-p_{i}$ and dilate by $\left|K_{i}\left(p_{i}\right)\right|^{-1 / 2}$ to get a new $A_{i}^{\prime}$. Note that the distance between boundary components of $A_{i}^{\prime}$ is $\geq \delta$. Also the absolute value of the curvature of $A_{i}^{\prime}$ is everywhere $\leq 1$ and is equal to 1 at the origin. Thus a subsequence of the $A_{i}^{\prime}$ will converge to an embedded minimal surface $M$ of finite total curvature and with curvature -1 at the origin. (See Section 2 of [15] for details of this kind of argument.) The surface 
is simply connected (because the intersection of $A_{i}$ with any ball of radius less than $r / 2$ is simply connected). Furthermore, the boundary is either

(1) empty,

(2) a straight line $L$, or

(3) two straight lines $L_{1}$ and $L_{2}$.

The first case does not occur because the only complete embedded simply connected minimal surface of finite total curvature is the plane. In case (2), $M$ lies in a halfspace (by (5.1)). By construction it actually lies to one side of a plane containing $L$. But now reflecting $M$ in $L$ gives a complete simply connected embedded surface of finite total curvature, which was already ruled out in case 1 . In case (3), $M$ lies in a cylinder around $L_{1}$ by (5.1) and so $L_{1}$ and $L_{2}$ are parallel. Using either catenoidal barriers or the halfspace theorem [6] one easily shows that $M$ is in fact the strip between $L_{1}$ and $L_{2}$. But this contradicts the fact that $M$ has curvature -1 at the origin. This proves that

$$
\left|K_{i}\left(p_{i}\right)\right|^{1 / 2}=o\left(1 / h_{i}\right)
$$

Now let $\phi_{i}$ be the first eigenfunction of the Jacobi operator on $A_{i}$, normalized to have a maximum value of 1 . Let $q_{i} \in A_{i}$ be the point where the maximum occurs. Of course $\phi_{i}$ is positive except at the boundary. Now translate $A_{i}$ by $-q_{i}$ and dilate by $h_{i}^{-1}$ to get a new annulus $A_{i}^{\prime}$. By (5.2), a subsequence of the $A_{i}^{\prime}$ converges to the strip between two parallel lines. By the Harnack inequality, a subsequence of the eigenfunctions converge to a nonnegative function $\phi$ on the strip that is 0 on the boundary and that attains a maximum value of 1 (at the origin). But $\phi$ is a nonnegative eigenfunction with eigenvalue $\leq 0$, or, in other words, a subharmonic function, and therefore cannot have an interior maximum.

Proof of Theorem 5.1. Let $\mathcal{M}$ denote the space of compact embedded minimal annuli with boundary curves in horizontal planes. Given $A \in \mathcal{M}$, let $z_{*}(A)$ and $z^{*}(A)$ denote the infimum and supremum, respectively, of the $x_{3}$-coordinate on $A$ (so that $A$ has boundary in $P_{z_{*}(A)} \cup P_{z^{*}(A)}$ ).

Let $X$ be a compact set of annuli in $\mathcal{M}$. We will show that $X$ can be 
deformed in $\mathcal{M}$ to a single annulus. First if, $f_{t}(A)=A \cap\left\{x \mid z_{*}(A)+t\left(z^{*}(A)-z_{*}(A)\right) \leq x_{3} \leq z^{*}(A)-t\left(z^{*}(A)-z_{*}(A)\right)\right\}$, then $f_{t}(0 \leq t \leq 1 / 3)$ deforms $X$ to a family $X^{\prime}$ of annuli with smooth boundary curves.

Now for $A \in X^{\prime}$, let $g_{t}(A)$ be obtained from $A$ by translating by $-t z_{*}(A) e_{3}$ and then dilating (about the origin) by $(1-t)+t /\left(z^{*}(A)-z_{*}(A)\right)$. Then $g_{t}(0 \leq t \leq 1)$ deforms $X^{\prime}$ to a family $X^{\prime \prime}$ of annuli with smooth boundary curves contained in the planes $x_{3}=0$ and $x_{3}=1$.

For $A \in X^{\prime \prime}$, let $\gamma_{0}^{A}$ be the component of $\partial A$ in $P_{0}$. Since the space of embedded planar curves is contractible, we can deform the family $\left\{\gamma_{0}^{A} \mid A \in\right.$ $\left.X^{\prime \prime}\right\}$ to the unit circle. That is, we can find $\gamma_{t}^{A}(0 \leq t \leq 1)$ so that $\gamma_{t}^{A}$ depends smoothly on $t$ and $\gamma_{0}^{A}$ and so that $\gamma_{1}^{A}$ is the unit circle for each $A$. It is convenient to define $\gamma_{t}^{A}$ to be $\gamma_{0}^{A}$ for $t<0$.

Now deform $X^{\prime \prime}$ to a new family $Y$ by the homotopy $[t, A] \mapsto A \cap\left\{x_{3} \in\right.$ $[0,1-t]\}$ for $0 \leq t \leq 1-h$ (where $h \in(0,1)$ will be determined shortly). Then $Y$ has boundary curves in $P_{0} \cup P_{h}$. Of course the homotopy does not affect the boundary curves in $P_{0}$.

Let $\gamma^{A, h}$ be the parametrization of $A \cap P_{h}$ of the form $\gamma^{A, h}(s)=\gamma_{0}^{A}(s)+h e_{3}+$ $\nu^{A, h}(s)$ where $e_{3}=(0,0,1)$ and $\nu^{A, h}(s)$ is a horizontal vector perpendicular to $\frac{d}{d s} \gamma_{0}^{A}(s)$.

Now consider the following family of pairs of curves:

$$
\Gamma_{t}^{A}=\left\{\begin{array}{ll}
\gamma_{0}^{A} \cup\left(\gamma_{0}^{A}+t e_{3}+(1-t) \nu^{A, h}\right) & 0 \leq t \leq 1 \\
\gamma_{(t-1)}^{A} \cup\left(\gamma_{(t-1)}^{A}+h e_{3}\right) & 1 \leq t \leq 2
\end{array} .\right.
$$

By the Lemma 5.2, there is an $\varepsilon>0$ such that $U_{\varepsilon}\left(\gamma_{(t-1)}^{A}\right)$ is mean convex and so that for $0<h<\varepsilon, \Gamma_{t}^{A}$ bounds no almost-stable minimal annuli embedded in $U_{\varepsilon}$. Fix an $h \in(0, \varepsilon)$ small enough so that $A_{0}^{\prime}=A_{0} \cap\{z \leq h\}$ lies in $U_{\varepsilon}\left(\gamma_{0}\right)$.

By the implicit function theorem (or, more specifically, the smooth dependence theorem (3.1) of [16]), for each $A \in Y$ we can lift the path $\Gamma_{t}^{A}$ to a path $\phi(t, A), 0 \leq t \leq b_{A}$, in $\mathcal{M}$ with $\phi(0, A)=A$ and $\partial \phi(t, A)=\Gamma_{t}^{A}$, and such that either

(1) $\phi(t, A)$ is stable for $t<b_{A}$ and almost-stable for $t=b_{A}$, or

(2) $b=2$ and $\phi(t, A)$ is stable for every $t \in[0,2]$. 
Now for $0 \leq t \leq b_{A}, \phi(t, A)$ is contained in $U_{\varepsilon}\left(\gamma_{(t-1)}\right)$. (This is because it is true for $t=0$ and therefore by the maximum principle true for every $t \in\left[0, b_{A}\right]$.) Thus (by the lemma) case (1) does not occur.

Now $\Gamma_{2}$ consists of two circles, so by [10], [14] or [13], it bounds at most one stable minimal annulus, namely a stable catenoid $C$. Thus $\phi$ is a homotopy from the inclusion $Y \rightarrow \mathcal{M}$ to the constant map $Y \rightarrow C$.

This proves that $\mathcal{M}$ is contractible.

Remark 5.1. We now make some remarks related to Theorem 6.1. Let $\mathcal{M}^{S}$ be the subset of $\mathcal{M}$ consisting of stable annuli, and $\mathcal{M}_{0,1}$ be the set of annuli in $\mathcal{M}$ with boundary curves in $P_{0} \cup P_{1}$. The argument above also shows that $\mathcal{M}^{S}$ is contractible. Moreover, $\mathcal{M}_{0,1}$ and $\mathcal{M}^{S} \cap \mathcal{M}_{0,1}$ are deformation retracts of $\mathcal{M}$ and $\mathcal{M}^{S}$, respectively, so they are also contractible. (As above, $g_{t}(0 \leq t \leq 1$ ) gives a deformation retraction of $\mathcal{M}$ to $\mathcal{M}_{0,1}$ and of $\mathcal{M}^{S}$ to $\mathcal{M}^{S} \cap \mathcal{M}_{0,1}$.)

Remark 5.2. The space of all smooth immersed minimal annuli with boundary in fixed distinct parallel planes breaks up into contractible components where two minimal annuli lie in the same component if and only if the winding number of their boundary curves are the same. This last statement follows directly from a straightforward adaptation of the proof of Theorem 6.1.

Remark 5.3. Consider the space $\mathcal{A}$ of embedded minimal annuli $A$ in $\mathbb{R}^{3}$ with a preferred boundary curve. $A$ can be deformed inside itself to a smooth very stable thin minimal ribbon and two such ribbon like minimal surfaces can be deformed to each other if and only if they are ambiently isotopic. A modification of the proof of Theorem 6.1 shows that each path component of $\mathcal{A}$ has the homotopy type of the space $\Gamma$ of isotopy classes of the knot represented by the preferred boundary curve of an $A \in \mathcal{A}$. The homotopy type of $\Gamma$ can be analyzed by applying the Smale Conjecture [3], which shows that the path components of $\mathcal{A}$ are homotopy equivalent to $S O(3)$. The path components themselves can be characterized in terms of framed knots.

Proof of Theorem 0.4. Recall the statement of Theorem 0.4 in the Introduction. Since every $A \in S$ can be deformed inside itself in $S$ to an example in $\mathcal{M}$, Theorem 5.1 shows $S$ is path connected, which proves Theorem 0.4. 
There are several possible other generalizations of Theorem 5.1. The most obvious one is to show that the corresponding space $\widetilde{\mathcal{M}}_{g}$ of genus- $g$ examples is path connected (or contractible). This problem is a special case of the following conjecture.

Conjecture 5.1. Suppose $\Sigma_{0}$ and $\Sigma_{1}$ are compact diffeomorphic embedded minimal surfaces with boundary in two parallel planes $P_{1}, P_{2}$. Further suppose that $\partial \Sigma_{0}$ is isotopic to $\partial \Sigma_{1}$ in $P_{1} \cup P_{2}$. If $\Sigma_{0}$ has exactly one boundary curve in $P_{1}$, then $\Sigma_{0}$ is isotopic to $\Sigma_{1}$ through embedded minimal surfaces $\Sigma_{t}$ with $\partial \Sigma_{t} \subset P_{1} \cup P_{2}$.

Remark 5.4. The above conjecture is motivated by Corollary 1 in [7]. This corollary states that if $\Sigma_{0}$ and $\Sigma_{1}$ satisfy the hypotheses of Conjecture 6.1, then they can be joined by a smooth isotopy $\Sigma_{t}, 0 \leq t \leq 1$, of embedded surfaces with $\partial \Sigma_{t} \subset P_{0} \cup P_{1}$ where the $\Sigma_{t}$ are contained in the slab between $P_{1}$ and $P_{2}$. Conjecture 6.1 fails when $\Sigma_{0}$ is allowed to have two boundary curves on $P_{0}$ and two boundary curves on $P_{1}$. See [7] for this example.

\section{REFERENCES}

1. Anderson, M., Curvature estimates for minimal surfaces in 3-manifolds, Ann. Scient. École. Norm. Sup. 18 (1985), 89-105.

2. Barbosa, J. L. and do Carmo, M. On the size of a stable minimal surface in $R^{3}$, Amer. J. Math. 98 (1976), no. 2, 515-528.

3. Hatcher, A. , A proof of the Smale Conjecture $\operatorname{Diff}\left(S^{3}\right) \simeq 0(4)$, Ann. of Math. 117 (1983), 553-607.

4. Hoffman, D. and Meeks III, W. H., The asymptotic behavior of properly embedded minimal surfaces of finite topology, J. Amer. Math. Soc. 2, no. 4.

5. _ A variational approach to the existence of complete embedded minimal surfaces, Duke Math. J. 57 (1988), no. 3, 877-894.

6. _ The strong halfspace theorem for minimal surfaces, Invent. Math. 101 (1990), 373-377.

7. Meeks III, W. H., The topological uniqueness of minimal surfaces in threedimensional Euclidean space, Topology 20 (1981), 389-410.

8. Meeks III, W. H. and Kuiper, N., Total curvature for knotted surfaces, Invent. Math. 77 (1985), 25-69.

9. Meeks III, W. H. and White, B., Minimal surfaces bounded by a pair of convex planar curves, Bull. of Amer. Math. Soc. 24 (1991), no. 1, 179-184, Research Announcement.

10. __ Minimal surfaces bounded by convex curves in parallel planes, Comment. Math.i Helv. 66 (1991), 263-278. 
11. Meeks III, W. H. and Yau, S. T., The classical Plateau problem and the topology of three-dimensional manifolds, Topology 21 (1982), no. 4, 409-442.

12. - The existence of embedded minimal surfaces and the problem of uniqueness, Math. Z. 179 (1982), 151-168.

13. Schoen, R., Uniqueness, symmetry, and embeddedness of minimal surfaces, J. of Differential Geom. 18 (1983), 791-809.

14. Shiffman, M., On surfaces of stationary area bounded by two circles, or convex curves, in parallel planes, Ann. of Math. 63 (1956), 77-90.

15. White, B., Curvature estimates and compactness theorems in 3-manifolds for surfaces that are stationary for parametric elliptic functionals, Invent. Math. 88 (1987), no. 2, 243-256.

16. White, B., The space of $m$-dimensional surfaces that are stationary for a parametric integrand, Indiana Univ. Math. J. 30 (1987), 567-602.

17. White, B., New applications of mapping degrees to minimal surface theory, J. Differential Geom. 29 (1989), 143-162.

UNIVERSITY OF MASSACHUSETTS, U. S. A.

StANFord UNIVERSity, U. S. A.

RECEIVED NOVEMBER 17, 1992. 